OAI-PMH: http://www.indteca.com/ojs/index.php/Revista Scientific/oai

Ensayo Original / Original Essay

\title{
Personas con Condiciones Especiales y su Sexualidad
}

Autora: Janeth Margarita Rodríguez Gómez Universidad de Carabobo, UC janethrodriguez 41@hotmail.com

Puerto Cabello, Venezuela

\section{Resumen}

Las personas que presentan alguna condición especial o dificultad para realizar a cabalidad sus funciones básicas, de aprendizaje, sociales, entre otras; requieren que se le respete su condición y que la triada escolar les proporcione todo tipo de información, más aún, información que le ayudara a ejercer una sexualidad sin conflictos internos. Conceptualmente las Necesidades Educativas Especiales están referida a una condición que presentan las personas y viene afectando algún aspecto en su vida, bien sea pedagógico, físico, o estructuralmente orgánico. El propósito del ensayo fue interpretar la sexualidad en los estudiantes con NEE para el desarrollo de sus potencialidades. En tal sentido, una necesidad educativa especial, representa todo aquello que es primordial para la adquisición de los objetivos o fines de la educación. Así pues, en resumidas cuentas, se busca con esta investigación que los estudiantes alcancen durante su escolaridad, el máximo crecimiento personal y social; en concordancia con el apoyo pedagógico, servicios o institutos educativos, que sean formados como seres responsables, protegidos ante el abuso y que expresen sus impulsos sexuales de la misma forma como se acepta socialmente la de otros, además que reciban educación sexual de profesionales o familiares.

Palabras clave: educación especial; sexualidad; medio familiar; comunicación. 


\title{
People with Special Conditions and their Sexuality
}

\begin{abstract}
People who have any special condition or difficulty to fully perform their basic functions, learning, social, among others; they require that their condition be respected and that the school triad provide them with all kinds of information, moreover, information that will help them to exercise a sexuality without internal conflicts. Conceptually, the Special Educational Needs are referred to a condition that people present and is affecting some aspect of their life, be it pedagogical, physical, or structurally organic. The purpose of the essay was to interpret sexuality in students with SEN for the development of their potential. In this sense, a special educational need represents everything that is essential for the acquisition of the objectives or aims of education. Therefore, in short, this research is aimed at students achieving maximum personal and social growth during their schooling; in accordance with pedagogical support, educational services or institutes, which are trained as responsible beings, protected from abuse and express their sexual impulses in the same way as others are accepted socially, in addition to receiving sexual education from professionals or relatives.
\end{abstract}

Keywords: special education; sexuality; family environment; communication. 


\section{Introducción al contexto de estudio}

El ensayo que a continuación se describe, viene a formar parte de diversos estudios que ha realizado la investigadora, ya que, desde la ejecución de su pregrado en el año 1995, hasta la actualidad, que está haciendo un postdoctoral, ha incursionado en la sexualidad escolar; puesto que, le llama la atención que al referirse a los aspectos sexuales, se ocultan o reprimen a los escolares, por ser temas para los adultos prohibidos, sin saber que ellos, al no encontrar respuestas acuden a vías o personas menos capacitadas para ofrecer información alguna, tal es el caso del entorno, compañeros, familiares o medios difusivos, que en vez de aclarar dudas distorsionan la información. Para emprender esta investigación y poder dejar claro al lector la importancia de tener presente que los temas sexuales desde estudios anteriores expresan tabúes y prejuicios sociales; más aún cuando se imparte a estudiantes con una condición especial.

Es evidente, que educar a personas con alguna condición especial conlleva a educar desde la igualdad, la equidad, la diferencia, el respeto, sus potencialidades, su cultura, su religión y sobre todo que no interfiera su necesidad educativa especial para formarlos en temas sexuales (Amorós y Pérez, 1993).

Las necesidades educativas especiales, vienen a representar una designación o nomenclatura que se les proporciona a los estudiantes en edad escolar, que por presentar diferentes formas temporal o parcial no se encuentran incursionando en escuelas de educación primaria, por tales razones, a dichas necesidades, se les enfatiza como educaciones individualizadas o especializadas; puesto que, los estudiantes requieren resolver conflictos de aprendizajes que por poseer una condición especial, no son capaces de lograr, por ende no son atendidos en educación primaria y se les ofrece en centros, servicios o institutos especiales que aseguran su prosecución escolar con personal capacitado para el logro académico de sus 
aprendizajes.

Las Necesidades Educativas Especiales (NEE), representa una condición que altera el ritmo de aprendizaje en los estudiantes, la misma ocasiona conflictos escolares que requieren de atención individualizada o específica durante su proceso escolar. Como afirman Marchesi, Coll y Palacios (2017): este término se aplica a los alumnos/as que presentan algún problema de aprendizaje a lo largo de su escolarización, problema que demanda una atención más específica y más recursos educativos que los que necesitan los compañeros de su edad.

Este término, data desde los años 70, ha sido utilizado de diferentes formas y ocasionado diferentes polémicas, por su excesiva profundidad y poca explicación. Del mismo modo, ha transitado por tres etapas o modelos según la conceptualización y política de educación especial médico-religiosa o filantrópica, medico-asistencial y el psicológico o escolar.

Una condición especial puede estar presente en las personas de manera temporal o definitiva, todo dependerá de la condición humana que tenga y su nivel escolar será determinado por la necesidad especial que presente tales como: retardo mental, síndrome de down, discapacidad visual, impedimento motor o físico, trastorno de hiperactividad, déficit de atención, trastorno espectro autismo, trastorno del lenguaje, problemas emocionales, dificultades de aprendizaje entre otros, las mismas puedan afectar el nivel del escolar y su adaptación al entorno.

Los escolares que presentan NEE, y son integrados en el sistema educación primaria, suelen padecer conflictos internos o bullying, ya que, no son bien vistos o aceptados, tanto por sus compañeros o maestros, en ocasiones se sienten fracasados repercutiendo en sus emociones. Los docentes que atienden a estudiantes con condiciones especiales, de igual forma sienten frustraciones ya que en su mayoría no poseen estrategias o herramientas adecuadas para impartir, de igual forma consideran que la vía 
más fácil es darle actividades sencillas, permitirle hacer lo que deseen, no exigirles como los demás estudiantes y pasarlos al grado inmediato superior. Por lo que, se cree que con estas acciones se están integrando al sistema educativo regular.

Las personas con NEE no deben ser objetos de lastima, deben ser tratado y respetados como todas las demás personas. Si aceptamos como maestro su integración, asumirla como tal, creer en ellos, resaltar sus potencialidades y tener la certeza que es un ser humano más en busca de aprender, recibir y dar. La responsabilidad de un docente es impartir conocimiento, conocer al grupo de estudiante, apoyarlos y no hacerlos sentir seres fracasados.

Ahora bien, cuando de educación sexual se trata, es importante ir más allá de lo académico, es ubicar al estudiante en su realidad, permitirle traer al aula de clase sus conflictos o inquietudes sexuales, para que cada uno enriquezca sus conocimientos a través de ellos y reflexionen al respecto, si el maestro no se siente conocedor del tema, debe valerse de especialistas en el área, ofrecer charlas con expertos en la materia y difundir terminologías apropiadas al aula o grado; puesto que, los espacios educativos son los que permiten realmente forman para la sexualidad.

Si los temas sexuales no se difunden a los adolescentes de manera clara, precisa y concreta, los llevan a buscar relaciones sexuales a temprana edad como inicio de su vida sexual activa. Exponiéndolos a enfermedades sexuales y a embarazos a temprana edad. Anteriormente, el transitar a la adolescencia era una etapa hermosa, única, apacible, con conflictos propios de ella, donde los cambios físicos se desarrollaban en su mayoría con normalidad. Hoy día se requiere dedicarle más comunicación y orientación.

Por lo que, la investigadora participe de escenarios educativos ha de preguntarse: ¿Cómo se imparte la sexualidad en estudiantes que presentan condiciones especiales? Mientras que el objetivo propuesto en esta 
investigación fue interpretar la sexualidad en los estudiantes con NEE para el desarrollo de sus potencialidades. Partiendo de un proceso metodológico de revisión hermenéutica hacia un enfoque interpretativo sobre la influencia del ámbito familiar o escolar en la sexualidad de los estudiantes con NEE, para luego relacionar el estudio con los esbozos teóricos que a continuación se presentan.

\section{Desarrollo Temático}

Para mantener una vida sexual activa, se hace necesario disfrutar del sexo, para que resulte placentero se deben romper barreras, tabúes y prejuicios; así como también se deben considerar posturas nuevas e innovadoras y dejar a un lado los miedos, vergüenzas o escrúpulos. Nunca permitan que estos conflictos sociales, por los valores inculcados de tus familiares te arruinen tu vida sexual activa.

Este ensayo reflejará algunos de los conflictos sexuales, los mismos pudiesen estar determinados en:

- Desagrado hacia el sexo oral.

- La higiene de los genitales.

- La masturbación de los órganos masculinos o femeninos.

- Satisfacción de las fantasías sexuales.

- Abandono de la zona de confort.

- Actividades sexuales rutinarias.

- Comunicación eficaz, constante y placentera.

- Mantenerse atractivo, sexy o sensual.

Por lo que, Cox sexóloga experta en el área (citada por Ramos 2016): recomienda, que durante las relaciones sexuales y para evitar en el acto 
sexual conflictos, deben olvidarse de los prejuicios y tapujos sociales, que realicen esta actividad como algo normal y hermoso de la vida, que se relajen y de una vez por todas, disfruten de una vida sexual a lo grande, no busquen en estas relaciones el perfeccionamiento y no permitan que el deseo se muera.

Díaz, (1999): considera la sexualidad más que sexo, no cree pertinente reducirla a los genitales, puesto que afecta la proyección de valores, su vida espiritual y el goce de la vida. Imposibilitando en los adolescentes sus posibilidades de expresión. Discurre el mismo autor, que se debe asesorar a los adolescentes y ofrecerles informaciones precisas para que su vida sexual adulta sea responsable, satisfactoria y sana.

En otro orden de ideas, es necesario acotar que en la actualidad, se está trabajando en las escuelas con las líneas intrasectoriales e intersectoriales, apoyados con especialistas tales como: orientadores, trabajados sociales, psicólogos y docentes; a manera de difundir la prevención de embarazo a temprana edad (PRETA), esta programación la están realizando profesionales pertenecientes al sistema educativo venezolano, siguiendo las líneas orientadoras del año escolar 2017-2018.

Al enunciar temas sexuales en las instituciones educativas, siempre estarán presentes las interrogantes en los estudiantes y es costumbre escuchar o visualizar en los pasillos: Hoy hablaran de sexo, risas, mejillas sonrosadas, vacilones, entre otras frases; así como también algunos términos como viagra, rapidito, besos, entre otros. Puesto que, con frecuencia estos temas de sexualidad no son comunicados frecuentemente en los centros educativos.

En tal sentido, se hace necesario que las escuelas sean las formadoras de los temas sexuales. Recientes estudios afirman que los jóvenes no son más activos hoy que hace 20 años atrás, de igual manera confirman que la información sobre sexualidad era recibida mayormente por revistas, compañeros o internet; siendo la escuela o los padres un porcentaje 
minoritario en la formación de estos temas sexuales; Continúan puntualizando los diferentes estudios, que si bien no se habla de sexo en los hogares, menos aún por parte de los maestros. En consecuencia, la educación sexual debería formar parte de la enseñanza escolar, debe proporcionarse de manera adecuada e incluir a los padres en esa formación y orientarlos para que aborden los temas en las casas y no espere a que otros la difundan.

Es importante mencionar, que al impartir educación sexual en las escuelas, debería ofrecerse de manera participativa, comunicativa o dialógica, la misma, debe generar debates o discusiones que permitan el esclarecimiento de las dudas si llegase a plantearse, dilucidar las diferencias de los puntos en común y plantear las vivencias sexuales. Se pretende que estos encuentros fortalezcan las comunicaciones, beneficien la reflexión colectiva de la vida sexual y faciliten las relaciones interpersonales entre los estudiantes que andan en busca de la promoción o proyección de los derechos sexuales 0 reproductivos; en pocas palabras, si es posible construir con la comunidad escolar análisis crítico o reflexivo de su comportamiento sexual y la de sus compañeros.

En el mismo orden de ideas, autores como Busquets, Cainzos, Fernández y Leal (1997): plantean que la sociedad específicamente los adolescentes, requieren ser escuchados y respetados, que se les otorgue el derecho de participar en la triada escolar, que sus actitudes por vivir la sexualidad sea aceptada y que se les permita defender sus propios derechos. Continúan expresando los actores mencionados, que la sexualidad abarca más que el acto sexual y la reproducción, ayuda a que las personas denoten sus sentimientos y emociones, les permite adquirir nuevos aprendizajes y decisiones necesarias para su vida adulta. Así como también, representa una energía fuerte de constante movimiento, hacia el deseo, el placer y la responsabilidad.

Hoy día, se imparte educación sexual, no se está en contra de ella, en 
lo que existe controversia, es al ser impartida en niños en edad preescolar o en estudiantes con condiciones especiales, lo que no se quiere es que sea una educación obligatoria para estas personas, ya que, nadie debe ser enfrentado, contra su voluntad, a contenidos relacionados con el sexo, y menos a tan corta edad o con una NEE. Exponen autores, que se están a favor de medidas educativas para luchar contra el abuso sexual, pero no de contenidos de educación sexual y sin ideología.

Es aquí la polémica al tema, ya que, de ser así muchos niños o personas especiales no recibirán ninguna educación sexual o exclusivamente aquella que les den sus padres, y se sabe que muchos padres no hablan de sexo con sus hijos o lo hacen de manera orientadora. Se cree erróneamente que al impartir sexualidad a esta comunidad preescolar o a la especial se le incita a tener relaciones a temprana edad, consideran los padres que por ser pequeños o especiales no tienen derecho a estar informados sobre su sexualidad y creen que aún están muy pequeños para manejar ciertas informaciones.

Un dato curioso, es que la educación sexual no se debe considerar igual que las otras maneras de aprender o de enseñar temas de otra índole, se cree que al impartir sexualidad se puede lastimar, herir u ofender con ello, ya que puede ser no apropiada para la edad preescolar o estudiantes especiales o con alguna discapacidad. Aun, no se tiene certeza quién saldría beneficiado con estos temas, se sugiere tener presentes los grados, aulas 0 niveles. No pareciera apropiado enseñar sobre sexualidad a los niños de corta edad o con NEE.

Según Foucault (2012): los temas sexuales están inmersos en la cultura de cada individuo, por estar sujeto a unas series de imposiciones relacionadas a la sexualidad, para él, el entorno (familia, escuela y comunidad), impone unas series de reglas y valores que se encuentran inmerso en la moralidad. Existen momentos que estas reglas y valores dependen exclusivamente de la 
enseñanza y estas a su vez se imparten de forma confusa. Por lo que, es aconsejables que los mecanismos que incitan hablar de sexo o sexualidad deben estar en las escuelas, son ellas quien conoce, dónde se habla, quien habla, como se habla y para quien se habla. Considera el mismo autor, que dependiendo de la cultura y del concepto que se tiene sobre la sexualidad, esta es la mejor forma de vigilar, supervisar y alertar.

Ahora bien, autor citado anteriormente hace énfasis al poder, ese poder represivo sobre el sexo, considera él, que el poder no siempre funciona así para las personas o maestros que trabajan con la sexualidad, lo importante del análisis de Foucault, es el de estar atentos al inducir, incitar o desviar. Por lo que, se hace menos difícil producir, ampliar y limitar los temas sexuales. Antes tales razones, el mismo autor asocia el poder para moldear cuerpos y mentes, y mantener supervisado el comportamiento mediante la familia, la escuela y la comunidad en general; es por ello, que enfatiza que el maestro o el psicopedagogo saben cómo conseguirlo.

En tal sentido, y por lo referido anteriormente es que se puede inferir que la población infantil y las que presentan alguna condición especial, enfrentan diversos conflictos influenciados por el adulto, la escuela y la comunidad, haciéndose necesario que este poder que representan los adultos debe estar inmerso en las vivencias de su sexualidad y no olvidar que la sexualidad no puede ser restringida ni privada ,sino difundida según sea su desarrollo evolutivo, pertinente a su edad y condición.

En el mismo orden de ideas, es de hacer énfasis que la sexualidad forma parte esencial de los seres humanos, por lo que, no se puede hablar de una sexualidad selectiva (adultos, padres, maestros, mayores, entre otros), la sexualidad es una sola. Tales son las circunstancias, de que si alguna persona presenta alguna condición especial o discapacidad requiere al igual que todas las personas, de expresar libremente su sexualidad, de vivir su experiencia amorosa, pero bajo la supervisión del adulto significativo. 
Cuando una persona posee alguna condición especial, se le debe tratar e informar tan igual como el que no la posee; es decir, que todas las personas gozan de deberes y derechos normados por leyes. Por lo que, se invita a respetarles su manera de actuar, pensar y expresar. Sin embargo, la población especial o con discapacidad presenta un nivel más elevado de riesgos relacionado al sexo o sexualidad, se hace necesario ofrecerles información sobre noviazgo, enfermedades de transmisión sexual, uso de preservativos, embarazos a temprana edad, entre otros. Si se educa esta población se tiene la certeza de abrir abanicos de oportunidades y su integración sería menos conflictiva.

Ahora bien, para la formación de las personas especiales existe un agente sumamente primordial, que son los padres y los representantes como figura familiar, es importante que compartan experiencias educativas 0 saberes cotidianos que le puedan ayudar $u$ orientar el manejo de la sexualidad de sus hijos; generalmente se les dificulta enfrentar los conflictos de índole sexual que atraviesan sus hijos y en especial, si es adolescentes. Se hacen renuente a ese tema tan transcendental de la vida, no consideran que sus hijos estén preparados sexualmente ni que tengan derecho de vivir una sexualidad a plenitud.

Parafraseando a Gogna, Adazko, Alonso, Binstock, Fernández, Pantelides, Portnoy y Zamberlin (2005): quienes consideran que comunicar es informar y estos términos tan primordiales, deben siempre estar presentes en la familia, más cuando de sexualidad se trata; padres e hijos tienen que conversar con frecuencia, plantearse inquietudes, intereses y necesidades sin tabúes ni prejuicios. Sin duda alguna que los temas sexuales no deben faltar en las comunicaciones familiares, deben manejar o enfrentar diferentes tópicos sexuales, considerando siempre en promover el dialogo entre ellos, donde cada uno es informante del otro. 


\section{Conclusiones}

Uno de los problemas actuales que enfrentan los adolescentes es la relación de familia, ya que se les dificulta comunicarse ambas partes, y más cuando esa comunicación es en función de la sexualidad o del sexo. Es importante que los adultos, maestros, especialistas o cualquier otro profesional que esté involucrado con la enseñanza de niños, niñas o adolescentes inicien el dialogo. Como adultos, cabeza de familia o responsables de velar por la educación de los niños, deberían iniciar encuentros comunicativos, con el fin de conocer, guiar, educar y orientar los aspectos relacionados al desarrollo sexual y sus posibles conflictos. Es por ello, que la mayoría los padres ocultan o esquivan conversaciones de este tipo, actuando como si los temas de sexo o sexualidad no existiera. Por lo que creen, que callar es mejor que conversar y más aun no darse por enterado de realidades sexuales.

Los padres no están preparados para asumir embarazos a temprana edad, puesto que para ellos su hija menor de edad no ha iniciado su vida sexual; cuando se enfrentan con estos conflictos sexuales, lo más razonable es el dialogo y enfrentar la situación con naturalidad, tranquilidad, sin recriminaciones ni amenazas, se debe tener presente que no será la primera ni la última que atraviesa por un embarazo, por lo que se recomienda mantener una comunicación fluida, que prevalezca la comprensión, el amor y la paz familiar, y no caer en el desespero (Atlantic Internacional University, 2013).

En tal sentido, se hace un llamado a padres y maestros a prevenir y para ello educar, educarse y orientar; pues es necesario enfrentar los riesgos a los que están sometidos sus hijos o estudiantes que al buscar información, esta es ofrecida de manera distorsionada, más aun si poseen una condición especial, o los que no poseen bases familiares sólidas.

Este ensayo va dirigido a los padres y representantes de adolescentes, para que se identifique con ellos, que comuniquen temas sexuales, puestos que son muy complejos y requieren ser abordados sin tapuje, tabúes, ni 
prejuicios. Que enfrenten su rol de padre y tengan el valor de conversar con sus hijos, o bien, que busquen ayuda profesional o inviten a sus hijos a consultar especialistas en el área sexual.

A manera de concluir, y poder llevar este mensaje investigativo, se hace un llamado en crear conciencia del papel preponderante que debe jugar la familia, escuela y la comunidad, como triada primordial, para rescatar el futuro de sus hijos o estudiantes, y de ver el nacimiento colectivo, basado en la solidaridad, en la realización de la individualidad y satisfacción racional de las necesidades reales del ser humano, para abrir paso al corazón de una sociedad feliz como está contemplado en el Proyecto Nacional "Simón Bolívar" (2007-2013).

\section{Referencias}

Amorós, A., \& Pérez, P. (1993). Por una educación intercultural. Madrid, España: Ministerio de Educación y Cultura.

Atlantic Internacional University (2013). Ensayo: Sexualidad en la adolescencia. La Romana, República Dominicana: Atlantic Internacional University. Recuperado de:

https://es.calameo.com/read/00523018866ffa056e469

Busquets, M., Cainzos, M., Fernández, T., \& Leal, A. (1997). Temas Transversais em Educação. Bases para uma formação integral. São Paulo: Ática.

Díaz, C. (1999). Sexualidad mucho más que sexo. Artículo publicado. Venezuela: Ediciones Aurora.

Foucault, M. (2012). Historia de la Sexualidad 2. El uso los Placeres. [Traducción de Martí Soler]. Madrid: Biblioteca Nueva, ISBN: 978-8415555-05-6, págs. 279. Recuperado de:

http://www.bibliotecanueva.es/admin/links/Historia\%20sex\%202.pdf

Gogna, M., (Coord.), Adazko, A., Alonso, V., Binstock, G., Fernández, S., 
Pantelides, E., Portnoy, F., \& Zamberlin, N. (2005). El embarazo y maternidad en la adolescencia: estereotipos, evidencias y propuestas para políticas públicas. 1a. ed. Buenos Aires, Argentina: CEDES, ISBN: 987-21844-2-9, págs. 344. Recuperado de: http://www.psi.uba.ar/academica/carrerasdegrado/psicologia/sitios cat edras/electivas/816 rol psicologo/material/unidad6/complementaria/e mbarazo maternidad adolescencia.pdf

Marchesi, A., Coll, C., \& Palacios, J. (2017). Desarrollo psicológico y educación: respuestas educativas a las dificultades de aprendizaje y del desarrollo. Colección: El Libro Universitario. Madrid, España: Alianza Editorial. ISBN: 978-84-9104-610-3. Recuperado de: https://www.alianzaeditorial.es/catalogos/capitulos promocion/LU0014 7801 9999971836.pdf

Proyecto Nacional "Simón Bolívar" (2007). Primer Plan Socialista (PPS) del Desarrollo Económico y Social de la Nación para el periodo 20072013. Caracas, Venezuela: Presidencia de la República Bolivariana de Venezuela. Recuperado de:

https://www.mppeuct.gob.ve/sites/default/files/descargables/proyectonacional-simon-bolivar.pdf

Ramos, A. (2016). Los 5 tabúes sexuales femeninos y con ellos disfrutar más. Alma, Corazón y Vida. El Confidencial. España: Titania Compañía Editorial, S.L. Recuperado de:

https://www.elconfidencial.com/alma-corazon-vida/2016-02-18/los-5tabues-sexuales-femeninos-y-que-hacer-con-ellos-para-disfrutarmas 1153970/ 


\section{Janeth Margarita Rodríguez Gómez \\ e-mail: janethrodriguez 41@hotmail.com}

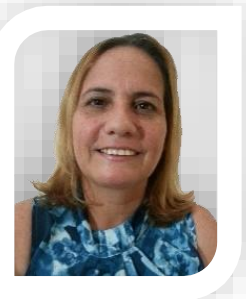

Nacida en Puerto Cabello, Estado Carabobo, Venezuela. Docente activa del Ministerio del Poder Popular para la Educación con 23 años a nivel de Primaria y Educación Especial. Experiencia laboral Universitaria: IUPMA, CUAM, UPEL, UNERS, UNA, ULAC, IUTEPAL y Misión Sucre. Estudios: Lic. En Educación, mención Educación Especial en la Universidad de Carabobo en Valencia. Maestría en Educación mención Educación Especial Integral en la Universidad Latinoamericana y del Caribe de Valencia, Dra. en Ciencias de la Educación de la ULAC. Investigadora y formadora de docentes. Asesora y Tutora de tesis a nivel de maestría y doctorado. Directora del Instituto de Educación Especial "Puerto Cabello". Árbitro de artículos. Línea de Investigación: Educación, Integración y Desarrollo Regional e Inclusión Social. 